\title{
On the Issue of Creating a Cream of Reparative Action for the Treatment and Prevention of Cheilitis
}

\author{
Kotenko Alexander, Yarnykh Tatyana, Zhivora Natalya, Rukhmakova Olga*, Kovalev Vladimir, Buryak Marina \\ Department of Drugs Technology, National University of Pharmacy, Kharkiv, Ukraine
}

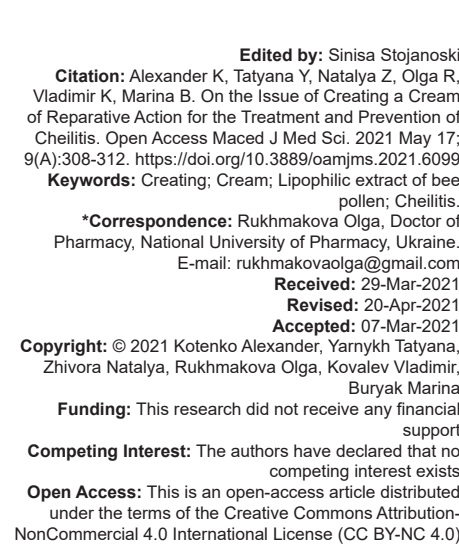

\begin{abstract}
BACKGROUND: Cheilitis is an inflammatory disease of the lips, its red border, mucous membranes, and skin There are meteorological, actinic, hypo-, and avitaminosis cheilitis, according to medical indications. Classification of cheilitis includes many types of diseases and is somewhat conditional. For these types of cheilitis treatment are most appropriate to use biologically active substances, in particular lipophilic extract of bee pollen. Lipophilic extract of bee pollen is a complex, concentrated plant-bee product with unique consumer and medicinal qualities. It should be noted that experiments have shown that the bee pollen lipophilic extract has a reparative effect higher than the widespread sea buckthorn oil.
\end{abstract}

AIM: The aim of the work is to create therapeutic and prophylactic cream with lipophilic extract of bee pollen for the cheilitis treatment and prevention.

MATERIALS AND METHODS: Cream preparation was conducted by phase inversion. Thermal and colloida stability, $\mathrm{pH}$ values, the quantitative content of carotenoids, viscosity, osmotic activity, and microbiological purity were determined by generally accepted methods.

RESULTS: Samples of the cream containing lipophilic extract of bee pollen are a homogeneous mass of soft consistency with light yellow color and pleasant specific odor. In estimating, the thermal stability cream samples cheilitis curing were successfully tested and proved to be stable. When centrifuging, the absence of stratification of the cream into fatty and aqueous phases after the test has showed the stability of the cream. The $\mathrm{pH}$ of the cream samples corresponds to the $\mathrm{pH}$ of the skin. During a qualitative test green color indicates the presence of carotenoids. Quantitative determination of the carotenoids amount in the cream for treatment and prevention cheilitis (in recalculation to $\beta$-carotene) corresponds to the set content of carotenoids in the cream of $5.4 \mathrm{mg}$. The viscosity of the developed cream at $16.2 \mathrm{~s}^{-1}$ is $68.38 \pm 0.21 \mathrm{~Pa} x \mathrm{~s}$, that fully corresponds to the viscosity of creams. In the study of osmotic activity, it was found that the cream with a glycerol content of $7 \%$ provides moderate osmotic activity of the drug for $8 \mathrm{~h}$, which may indicate the absence of overdrying and irritating effect. In terms of microbiological purity, the investigated cream with lipophilic extract of bee pollen meets the requirements of the State Pharmacopoeia for finished medicines for topical use (category 2).

CONCLUSION: For the cheilitis treatment and prevention has suggested the composition of the cream which contains biologically active substance - lipophilic extract of bee pollen. The technology of the cream with lipophilic extract of bee pollen was developed. Thermal and colloidal stability, $\mathrm{pH}$ values were studied; the quantitative content of carotenoids, viscosity, osmotic activity, and microbiological purity was determined. The research carried out made it possible to make sure of the correctness and rationality of the developed technology of the cream for the treatment and prevention of cheilitis.

\section{Introduction}

A sharp change in living conditions is a challenge for any creature, especially for its outer shells. Concerning humans, we are primarily talking about the skin and mucous membranes of the eyes, nose and in particular the lips their red border and mucous membranes. It should be noted that the lips were among the first to deal with the weather adversity and other negative effects of environmental influences. In addition, the lips are on the border of the inner state of human and his environment more than any other organ. Summarizing aforesaid, we can say that almost everyone at least once in their life has experienced inflammation of the lips and its mucous membranes.
Inflammatory disease of the lips, its mucous membranes and red border are called cheilitis. Considering that pathological condition is very common and many clinical forms of cheilitis are combined with each other, the classification of cheilitis is quite complex and somewhat conditional [1], [2], [3].

First of all, there is meteorological cheilitis, which is formed under the harmful influence of weather conditions. Actinic cheilitis is similar to the meteorological, but as an etiological component has primarily a harmful effect of sunlight [4], [5]. At first glance, it seems that these two types of cheilitis are the same, but they differ slightly in the manifestations, location, and course of the disease, even if both have no complicating factors. Sometimes meteorological and actinic cheilitis are supplemented by allergic cheilitis, 
which as an etiological factor has an allergic reaction to any irritating agent in the environment [6]

Hypo- and avitaminosis cheilitis based on poor nutrition along with difficult working conditions is quite common.

Herpetic and fungal cheilitis has infectious origin and is treated with antimicrobial agents. The etiological component of exfoliative cheilitis is uncomplicated vegetoneurotic conditions [7], [8]. In contrast to cheilitis caused by weather conditions, which are more common for men, exfoliative cheilitis is observed primarily for girls and young women. Of course, as part of a comprehensive treatment should not be used only skin care products but also psychotherapy and drugs that have a calming effect and regulate neurotic states.

It is clear that the most common among the population of Ukraine are uncomplicated forms of cheilitis: meteorological, actinic, hypo-, and beriberi cheilitis. In case of incongruous aid, the situation can become significantly more complicated, cheilitis from a small cosmetic problem can become a serious medical problem, the inflammation becomes more widespread, and causes pain and suffering to patients [9], [10].

Therefore, the aid in the early stages of treatment should be properly safe and effective. For this reason, as an active ingredient for medicines attract the attention of biologically active natural complexes with emollient, anti-inflammatory, and reparative effects and rich in vitamins necessary for the skin.

The use a number of natural origin substances with vitamin activity hass more pronounced biologically active effect in a composition of a single-drug than when the same substances are used separately. In this case, the lipophilic extract of bee pollen containing a complex of carotenoids, tocopherol matches, and unsaturated fatty acids, which are important for the cheilitis treatment. Given that, as shown above, poor nutrition and vitamin deficiency play a huge role in the occurrence of uncomplicated forms of cheilitis; therefore, offered composition is very appropriate.

Lipophilic extract of bee pollen is a complex, concentrated plant-bee product with unique consumer and medicinal qualities. It should be noted that experiments have shown that the bee pollen lipophilic extract has a reparative effect higher than the widespread sea buckthorn oil [11]. The objective of this work is to substantiate the composition and technology of therapeutic and prophylactic cream with bee pollen lipophilic extract for the cheilitis treatment.

\section{Materials and Methods}

The preparation of the cream was carried out by the conventional phase inversion method as follows.
Preparation of the oil phase: With Vaseline oil at a temperature of up to $65-70^{\circ} \mathrm{C}$ fused stearin and sodium hydroxide and left for 15-20 min for neutralization; then added glycerol monostearate, synthetic alcohols, butyloxyanisole, and lipophilic extract of bee pollen.

Simultaneously, the purified water with glycerin was heated up to $65-70^{\circ} \mathrm{C}$ temperature and dissolved bronitrol. With the stirrer running, to the oil phase portion wise was added aqueous phase and emulsified using micro-shredder tissue at 3000 rpm, followed by artificial cooling.

The obtained cream samples were tested for colloidal and thermal stability, $\mathrm{pH}$, qualitative and quantitative composition of carotenoids, viscosity, osmotic activity, and microbiological purity.

Colloidal stability was estimated after centrifugation (6000 rpm; $5 \mathrm{~min}$, temperature $45 \pm 2^{\circ} \mathrm{C}$ ).

Thermostability determined in the absence of cream stratification in the study at different temperatures modes: 7 days in an incubator at $40 \pm 2^{\circ} \mathrm{C}$, then 7 days in a refrigerator at $10 \pm 2^{\circ} \mathrm{C}$ and 3 days at $20 \pm 2^{\circ} \mathrm{C}$. Samples were considered stable if they remained homogeneous after thermal or mechanical exposure [12].

$\mathrm{pH}$ determination. $2.5 \mathrm{~g}$ of cream was placed in a beaker with a capacity of $100 \mathrm{~mL}, 50 \mathrm{~mL}$ of water $\mathrm{R}$ was added and stirred with a glass rod for $10 \mathrm{~min} ; \mathrm{pH}$ determined potentiometric method according to SPU 2.0.

Identification of carotenoids: $0.5 \mathrm{~g}$ of cream was placed in a separatory funnel with a ground stopper, 3 $\mathrm{mL}$ of chloroform $R$, a few crystals of sodium chloride $R$ were added and mixed thoroughly. The chloroform layer was poured into a dry tube with a ground stopper and $2 \mathrm{~mL}$ of antimony (III) chloride solution $R$ was added; green color was observed [13].

Quantitative determination of the carotenoids amount: Nearly $1.0 \mathrm{~g}$ (exact portion) of the cream was placed in a beaker, dissolved in $30 \mathrm{~mL}$ of a mixture of $96 \%$ alcohol $R$ - chloroform $R(1: 1)$, quantitatively transferred to a volumetric flask with a capacity of 50 $\mathrm{mL}$, adjusted the volume of the solution to the mark with the specified solvent mixture and stirred [13]. Absorbance resulting solution was measured on a spectrophotometer for the lengths of a wave of 450 $\mathrm{nm}$ in the cell with a layer thickness of $10 \mathrm{~mm}$, using as comparison mixture solution of $96 \%$ alcohol $R-$ chloroform $R(1: 1)$. In parallel, the optical density of the standard solution of potassium dichromate solution was measured. Water $R$ was used as a reference solution.

Viscosity was determined on a Brookfield DV-II + PRO viscometer (USA) using a special rotary adapter, which allows rheological studies with a minimum amount of sample $(25.0 \pm 0.5 \mathrm{~g})$. The spindle of the SC4-21 brand was used. Structural and mechanical studies were performed at certain temperatures, which were recorded by a sensor connected to the sample chamber. 
Study of osmotic properties: The kinetics of water absorption was determined in in vitro experiments by dialysis through a semipermeable membrane company "Hoechst" at a temperature of $37 \pm 0.1^{\circ} \mathrm{C}$ by changing the mass of the chamber with the sample. To evenly distribute the sample on the membrane, it was pre-melted at a temperature of $\left(37-38^{\circ} \mathrm{C}\right)$ and applied in a uniform layer on the surface of the membrane. Then, the inner container together with the test sample was placed in a dialysis chamber, in which the calculated amount of water was poured in advance. Dialysis was performed with constant stirring with a magnetic stirrer. The mass of the inner container was measured at regular intervals (1 h) until a constant mass was established on five parallel determinations. The difference between the obtained results was used to determine the amount of absorbed water.

Studies of microbiological purity were conducted on the basis of the State Institution "Mechnikov Institute of Microbiology and Immunology" (Ukraine).

All results of pharmaco-technological, physicochemical and microbiological studies were subjected to statistical processing.

\section{Results}

The selection of excipients for the base composition of the cream containing a lipophilic extract of bee pollen was done purposefully with the end result of the development of an emulsion base of the first type. Vaseline oil was selected as the oil phase and solvent for the lipophilic extract of bee pollen in the cream due to its chemical nature - chemical inertness, storage stability, and compatibility with all types of emulsifiers. In addition, mineral oil promotes the formation of a semipermeable film on the skin, which prevents water from evaporating from the skin surface. The concentration of Vaseline oil was $20 \%$.

The stability, high dispersion, and the required rheological properties of emulsions provide using of the first and second type emulsifiers mixture. When stabilizing oil/water emulsions with an emulsifier of the first type in combination with higher fatty alcohols of the $C_{16}-C_{18}$ fraction are formed emulsions with viscousplastic properties of oily consistency.

As an emulsifier of the first type for the base selected stearin, for partial saponification it was neutralized by sodium hydroxide. As the second type, emulsifier used synthetic primary alcohols fraction of $\mathrm{C}_{16}-\mathrm{C}_{18}$ and glycerol monostearate. The research was carried out and shown that they have the highest viscosity at ratio of components $1: 1$, that is, $3.0 \%$ glycerol monostearate and $0.8 \%$ synthetic alcohols
$\mathrm{C}_{16}-\mathrm{C}_{18}$. In addition, $7.0 \%$ glycerol was added to the composition of emulsion to increase the emulsion viscosity and ensuring frost resistance [14].

Bee pollen lipophilic extract was administered to the dosage form in a concentration from $1.0 \%$ to $10.0 \%$ of the substance, after that restorative and anti-inflammatory activity was studied [11]. Preclinical studies of ointments with bee pollen lipophilic extract in the ointment showed that it has pronounced antiinflammatory and reparative activities at a $3.0 \%$ concentration in the ointment [15]. Samples screening with different quantitative content of lipophilic extract of bee pollen carried out on the models of aseptic skin ulcers in rats (the study of antialterative activity), linear cut wounds in rats (the study of reparative activity), and thermal burns in mice (the study of anti-inflammatory activity).

Based on the studies, the effective concentration of lipophilic extract of bee pollen in the cream was determined $-3.0 \%$ [11]. Preclinical studies of the cream samples with lipophilic extract of bee pollen were carried out and shown that they have pronounced anti-inflammatory and wound-healing activities with $3.0 \%$ lipophilic extract of bee pollen concentration [15].

Thus, there is reason to believe that the concentration of $3.0 \%$ for lipophilic extract of bee pollen is the best and most balanced, which was taken as a sample for preparing health-care cream cheilitis curing.

Chain autocatalytic nature of chemical processes of carotenoid oxidation, accelerating reactions in solution caused necessity for chemical stabilization of carotenoids in the medicine. Therefore, for the composition of the cream for cheilitis curing proposed to administrate butyloxyanisole in our chosen concentration $0.15 \%$ based on research, as a preservative bronitrol was selected [14]. Thus, the cream composition for the cheilitis prevention and treatment is shown in Table 1.

Samples of the cream for the cheilitis prevention and treatment containing lipophilic extract of bee pollen are a homogeneous mass of soft consistency with light yellow color and pleasant specific odor. In estimating, the thermal stability cream samples cheilitis curing were successfully tested and proved to be stable at temperatures $40^{\circ} \mathrm{C}, 5^{\circ} \mathrm{C}$, and $20^{\circ} \mathrm{C}$. When centrifuging, the absence of stratification of the cream into fatty and

Table 1: Composition of the cream with bee pollen lipophilic extract

\begin{tabular}{ll}
\hline Purpose of the component & Cream components, content in \% \\
\hline Disperse phase & \\
Reparative, anti-inflammatory action & Lipophilic extract of bee pollen 3.00 \\
Oil phase & Vaseline oil 20.00 \\
Antioxidant & Butyloxyanisole 0.15 \\
Emulsifiers & Stearin 4.00 \\
& Sodium hydroxide 0.15 \\
& Glycerol monostearate 3.00 \\
& Synthetic alcohols $\mathrm{C}_{16}-\mathrm{C}_{18} 0.80$ \\
Dispersion medium & Purified water 61.80 \\
Aqueous phase & Glycerin 7.00 \\
Preservative & Bronitrol 0.10 \\
\hline
\end{tabular}


Table 2: Stability investigation of the cream with lipophilic extract of bee pollen

\begin{tabular}{|c|c|c|c|c|c|c|c|c|c|c|c|}
\hline $\begin{array}{l}\text { No. of } \\
\text { sample }\end{array}$ & Appearance & Color, odor & $\begin{array}{l}\text { Colloidal } \\
\text { stability }\end{array}$ & $\begin{array}{l}\text { Thermal } \\
\text { stability }\end{array}$ & $\begin{array}{l}\mathrm{pH} \text { of the aqueous } \\
\text { dispersion, from } \\
5.50 \text { to } 6.50\end{array}$ & $\begin{array}{l}\text { Identification of } \\
\text { carotenoids }\end{array}$ & $\begin{array}{l}\text { Quantitative } \\
\text { definition, not } \\
<5.4 \mathrm{mg}\end{array}$ & $\begin{array}{l}\text { Viscosity at } \\
16.2 \mathrm{~s}^{-1} \\
\mathrm{~Pa} \times \mathrm{s}\end{array}$ & $\begin{array}{l}\text { Osmotic } \\
\text { activity, } \\
\%\end{array}$ & Microbiological purity & $\begin{array}{l}\text { Shelf life, } \\
\text { months }\end{array}$ \\
\hline 111018 & $\begin{array}{l}\text { Homogeneous } \\
\text { cream of soft } \\
\text { consistency }\end{array}$ & $\begin{array}{l}\text { Light-yellow, } \\
\text { specific } \\
\text { pleasant }\end{array}$ & Stable & Stable & $5.80 \pm 0.12$ & Green color & $5.82 \pm 0.15$ & $68.38 \pm 0.21$ & 65.8 & $\begin{array}{l}\text { CFU in } 1.0 \mathrm{~g} \text { bacteria } \\
\text { and fungi total no } \\
\text { more than } 10^{2}\end{array}$ & Initial sample \\
\hline 111018 & $-1 /-$ & $-1 /-$ & $-1 /-$ & $-11-$ & $5.85 \pm 0.13$ & $-1 /-$ & $5.74 \pm 0.17$ & $69.12 \pm 0.18$ & 63.4 & $-1 /-$ & 6 \\
\hline 111018 & $-11-$ & $-1 /-$ & $-1 /-$ & $-1 /-$ & $5.88 \pm 0.13$ & $-1 /-$ & $5.72 \pm 0.15$ & $69.21 \pm 0.23$ & 64.1 & $-1 /-$ & 12 \\
\hline 111018 & $-\| /-$ & $-\| /-$ & $-\| /-$ & $-\| /-$ & $5.81 \pm 0.12$ & $-1 /-$ & $5.65 \pm 0.19$ & $69.13 \pm 0.15$ & 63.6 & $-1 /-$ & 18 \\
\hline 111018 & $-\| /-$ & $-1 /-$ & $-1 /-$ & $-\| /-$ & $5.90 \pm 0.11$ & $-11-$ & $5.58 \pm 0.14$ & $68.95 \pm 0.19$ & 62.8 & $-1 /-$ & 24 \\
\hline 111018 & $-1 /-$ & $-1 /-$ & $-1 /-$ & $-1 /-$ & $5.88 \pm 0.13$ & $-1 /-$ & $5.56 \pm 0.15$ & $69.08 \pm 0.20$ & 63.1 & $-\| /-$ & 27 \\
\hline
\end{tabular}

$n=5, p=0.95$; packing: tubes of cream of $30 \mathrm{~g}$

aqueous phases after the test has showed the stability of the cream. Conducted results of the analysis at room temperature have showed the stability of the cream with lipophilic extract of bee pollen.

The results of the analysis are given in Table 2.

As can be seen from the table, the $\mathrm{pH}$ of the cream samples for treatment and prevention cheilitis corresponds to the $\mathrm{pH}$ of the skin. During a qualitative test green color indicates the presence of carotenoids. Quantitative determination of the carotenoids amount in the cream for treatment and prevention cheilitis (in recalculation to $\beta$-carotene) corresponds to the set content of carotenoids in the cream of $5.4 \mathrm{mg}$.

The viscosity of the developed cream at $16.2 \mathrm{~s}^{-1}$ is $68.38 \pm 0.21 \mathrm{~Pa} x \mathrm{~s}$, that fully corresponds to the viscosity of creams.

In the study of osmotic activity, it was found that the cream with a glycerol content of $7 \%$ provides moderate osmotic activity of the drug for $8 \mathrm{~h}$, which may indicate the absence of overdrying and irritating effect.

In terms of microbiological purity, the investigated cream with lipophilic extract of bee pollen meets the requirements of the State Pharmacopoeia for finished medicines for topical use (category 2).

The research carried out made it possible to make sure of the correctness and rationality of the developed technology of the cream for the treatment and prevention of cheilitis.

The patent of Ukraine for the invention was received for the cream composition [16].

\section{Conclusions}

For the cheilitis treatment and prevention have suggested the composition of the cream which contains biologically active substance - lipophilic extract of bee pollen. The technology of the cream with lipophilic extract of bee pollen was developed. Thermal and colloidal stability, $\mathrm{pH}$ values were studied; the quantitative content of carotenoids, viscosity, osmotic activity, and microbiological purity were determined. The research carried out made it possible to make sure of the correctness and rationality of the developed technology of the cream for the treatment and prevention of cheilitis.

\section{References}

1. Lugović-Mihić L, Pilipović K, Crnaric I, Šitum M, Duvančić T. Differential diagnosis of cheilitis - How to classify cheilitis? Acta Clin Croat. 2018;57(2):342-51. https://doi.org/10.20471/ acc.2018.57.02.16

PMid:30431729

2. Lutskaya I. Diseases of the lips - cheilitis. Dermatology 2016;1:58-62.

3. Averyanov S, Romeiko I. Results of approbation of ointments for the treatment of cheilitis. Mod Probl Sci Educ. 2015;3:217-24.

4. Khismatullina Z, Bulgakova A, Khamzina G, Zatsepina M. Care for diseases of the red border of the lips. Clin Dermatol Venerol. 2018;17(2):81-6. https://doi.org/10.17116/ klinderma201817281-86

5. Bertini F, Sgarbi F, Tera T, Brandão A, Cavalcante A. Histological analysis of the actinic cheilitis: An interobserver approach. Int $J$ Dent. 2010;9(1):6-10.

6. O'Gorman S, Torgerson R. Contact allergy in cheilitis. Int $\mathrm{J}$ Dermatol. 2016;55(7):386-91.

7. Girijala R, Falkner R, Dalton S, Martin B. Exfoliative cheilitis as a manifestation of factitial cheilitis. Cureus. 2018;10(5):e2565. https://doi.org/10.7759/cureus.2565 PMid:29974020

8. Rabinovich O, Rabinovich I, Umarova K, Denisova M. Modern possibilities of cheilitis treatment. Clin Dent. 2016;3:36-8.

9. Dancyger A, Heard V, Huang B, Suley C, Tang D, Ariyawardana A. Malignant transformation of actinic cheilitis: A systematic review of observational studies. J Investig Clin Dent. 2018;9(4):e12343. https://doi.org/10.1111/jicd.12343 PMid:29863311

10. Ariotti C, Gabriel A, Ribeiro J, Martins M, Vargas P, Pilar E, et al $\mathrm{PI} 3 \mathrm{~K} / \mathrm{AKT} / \mathrm{mTOR}$ pathway activation in actinic cheilitis and lip squamous cell carcinomas. J Eur Acad Dermatol Venereol. 2020;34(9):505-6. https://doi.org/10.1111/jdv.16420 PMid:32255224

11. Yakovleva L, Tkacheva O, Kotenko O. Pharmacological study of a new reparative agent with bee pollen. News Pharm. 1998;1:86-8

12. State Consumer Standard of Ukraine. Cosmetic Creams General Technical Conditions. DSTU 4765-2007. Kiev: State Consumer Standard of Ukraine; 2008. p. 7.

13. Kotenko O, Khanin V, Tikhonov O, Zhivora N, Yu A. Standardization and study of the stability of the ointment 
"Lipovit". News Pharm. 2012;4:43-6.

14. Kotenko O, Tikhonov O, Zhivora N. Development of the composition of therapeutic and prophylactic cream for dry skin. News Pharm. 2007;2:33-8.

15. Korzh M, Goridova L, Tikhonov O, Zhivora N, Tkacheva O. Clinical trials of Lipovit ointment. Clin Pharm. 2001;5(1):48-51.
16. Kotenko O, Yarnykh T, Zhivora N, Rukhmakova O. Patent of Ukraine for a Utility Model No. 142983 dated 10.07.2020, Bull. No. 13 on the Issuance of IPC Patent (2020.01): A61K 9/06, A61K 31/00, A61P 17/00. Therapeutic Cream for the Treatment and Prevention of Meteorological, Actinic and Hypovitaminosis Cheilitis. Ukraine: International Patent Classification; 2020. 